\title{
FINE STRUCTURE OF SOLAR MAGNETIC FIELDS
}

\author{
HAROLD ZIRIN
}

Big Bear Solar Observatory, Hale Observatories, California Institute of Technology, Carnegie Institute of Washington

(Received 20 July, 1971)

\begin{abstract}
The deduction of magnetic fields from chromospheric structure is extended to active regions and transverse fields. Fields independently predicted by these rules from a high resolution $\mathrm{H} \alpha$ filtergram are compared with a high resolution magnetogram. The $\mathrm{H} \alpha$ method has the advantage over conventional magnetograms that it shows transverse fields and relates the fields to the real Sun. It has the disadvantage that higher spatial resolution is required and that it is difficult and time consuming in very complicated regions.

The response of the chromosphere to magnetic fields is most consistent. Vertical field is invariably marked by bright plage, with brightness roughly proportional to the field strength (except for sunspots). All dark tibrils mark transverse fields and are parallel to field lines. All polarity changes are marked by dark fibrils, which may be transverse fibrils perpendicular to the field boundary, or filaments (prominences) which connect more distant points, and in which the field lines run nearly parallel to the boundary. The asymmetry between preceding and following polarity found by Veeder and Zirin (1970) does not exist; it was due to the low resolution of the Mount Wilson magnetograms.

The complexity of active region field structure depends on the history of the region; all flux erupts in simple bipolar form, and lines of force remain connected to sibling spots until reconnection takes. place. Thus the complex structure only occurs after eruption of several dipoles which reconnect. The phenomenon of 'inverted polarity' turns out to be due to the emergence of satellite bipolar fields, where the $p$ spot merges with the rest of the $p$ field and the $f$ spot appears as an included $f$ field. Flares usually occur when the field lines from $f$ spot reconnect from its sibling to the main spot.
\end{abstract}

\section{Introduction}

It was shown by Veeder and Zirin (1970) that chromospheric morphology could be used to deduce the distribution of weak magnetic fields on the surface of the Sun. This work was limited by the resolution of the pictures with the five inch Caltech photoheliograph $\left(2^{\prime \prime}\right)$ and the Mt. Wilson daily magnetograms $\left(17^{\prime \prime}\right)$. With improved 2 " magnetograms from Aerospace Corporation and Kitt Peak the relations are more clear (Zirin, 1970a, b). For each improvement, a few pairs of filtergrams and magnetograms become a 'Rosetta Stone' to determine the relationships involved, which then can be applied to the millions of individual filtergrams to deduce the fields when no magnetograms were available.

In this paper we summarize relationships deduced from new high resolution $\mathrm{H} \alpha$ pictures and then test them by comparing the predicted magnetic field with a high resolution magnetogram. We then apply the method to understanding the structure and history of several active regions.

The value of comparison of $\mathrm{H} \alpha$ and magnetic fields lies beyond the simple production of magnetograms; it lies in the evaluation of the chromospheric morphology, in the understanding of the development of solar magnetic fields and in the possibility of the assessment of the physical structure of the different magnetic regions. 
We find that the history of any field line plays an important role in its shape; until reconnection takes place, bipolar fields (and there are no monopolar fields on the Sun) remain connected by field lines, often over great distances. Because the $\mathrm{H} \alpha$ pictures enable us to evaluate the direction and strength of transverse fields, they enable us to trace some of these lines of force and infer others.

Our basic data are filtergrams from the Big Bear Solar Observatory, made with twin ten inch $f / 14$ lenses, with secondary magnification to produce a solar image between three and six inches in diameter. With SO 392 film the modulation appears to be about $25 \%$ at 1 arc sec and $10 \%$ at one $\frac{1}{2}$ arc sec these are guesses: A new Halle filter using a contrast element of $0.7 \AA$ (this change was proposed to Bernhard Halle Nachfl. by the author as a result of calculations of the optimum sideband suppression for $\mathrm{a} \frac{1}{2} \AA$ filter; I am greatly indebted to them for carrying out this improvement) made possible greatly improved contrast in $\mathrm{H} \alpha$; because the resolution depends on the contrast, the limiting resolution is improved. Thus two systems operate simultaneously in various pairs of wavelengths. The pictures are compared with longitudinal magnetograms; by added assumptions the vector field may be deduced.

The higher spatial resolution of the Big Bear pictures makes it possible to recognize the response of chromospheric fine structure to the governing magnetic fields. Since the identification of a chromospheric structure requires an association of several picture elements, the resolution possible is somewhat reduced by comparison with the magnetograph, which measures the Zeeman effect in a single point. We assume the magnetograms are always right and disagreements are due to poor deduction or incorrect rules.

\section{Rules for Deduction of the Magnetic Field}

To illustrate the rules which are a synthesis of previous and present work, we have used pictures taken October 11, 1970 of a fairly simple region, Mt. Wilson $18157+$ +18161 , marking numbers on Figure $1(\mathrm{H} \alpha)$ and $2(\mathrm{H} \alpha+0.5 \AA)$ to indicate different reatures of interest.

A. Dominant preceding sunspots $(1 p, 2 p)$ are identified by large size and welldeveloped penumbras. Of course, they normally precede, but there are occasional exceptions. Dominant following spots ( $1 f$ ) usually follow $p$ spots, do not have well developed penumbral structure, and are of course separated from the $p$ spots by field transitions.

B. All regions of vertical field ( $3 p$ and $3 f$ ) except sunspots are bright in $\mathrm{H} \alpha$ and have fine granular structure at $\pm \frac{1}{2} \AA$. The brighter the plage, the greater the field strength. C. Emerging flux regions (Weart and Zirin, 1969) or EFR are always marked by bright plage, crossed by dark arches (Bruzek, 1967) $5 p$ and $5 f$ mark an EFR arising in an existing $f$ plage. The EFR's are invariably symmetric and bipolar.

D. All regions of predominantly transverse field are dark. All dark fibrils are parallel to the magnetic lines of force. Older neutral lines (4) inside the active region are marked by what Prata (1971) calls field transition arches (FTA) parallel to field lines 


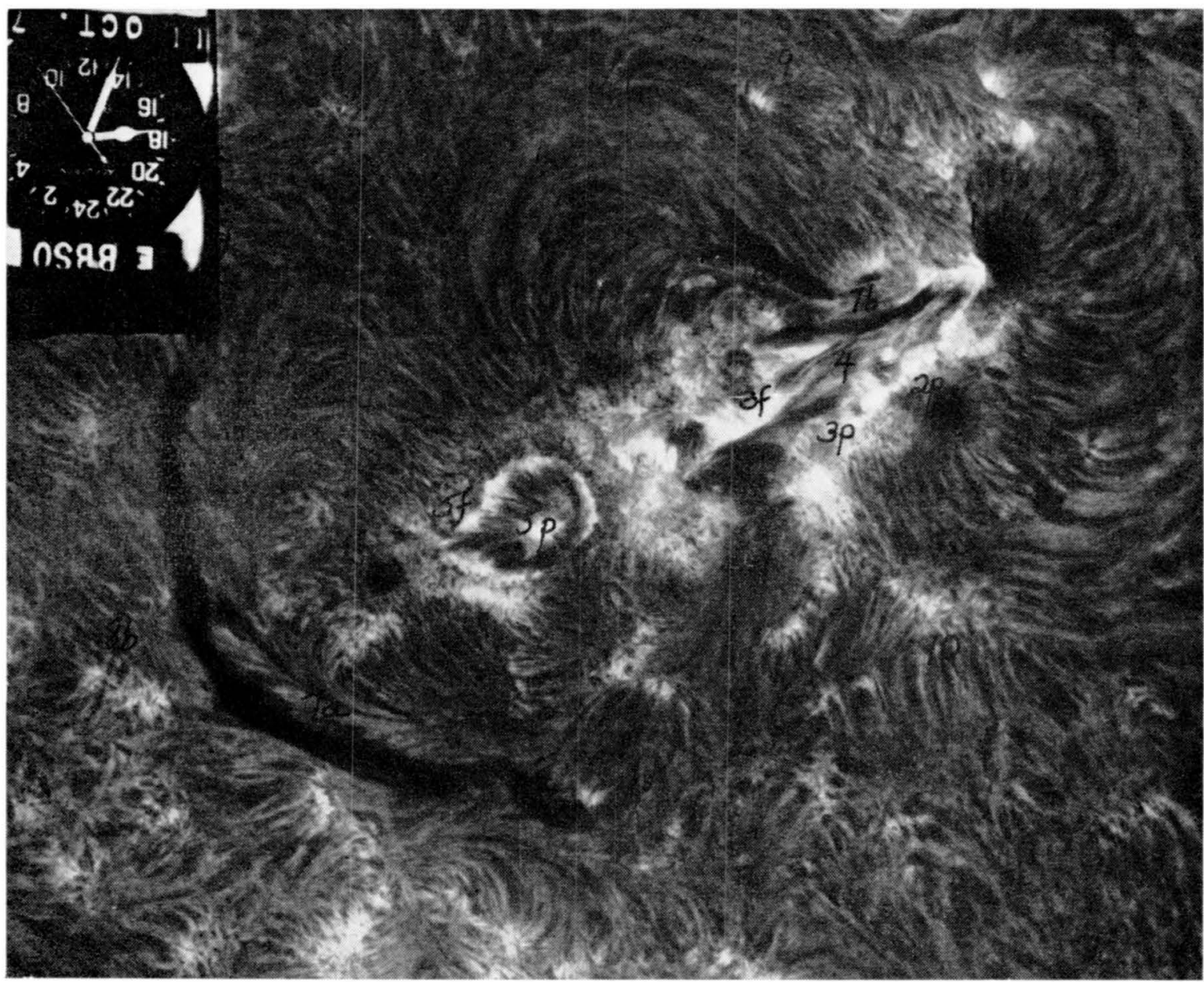

Fig. 1. Mt. Wilson 18157 and 18161, 1802 UT, October 11, 1970, H $\alpha$ center line, $\mathrm{N}$ top, E left (the clock is in the NE corner of all Big Bear photos). All photos made with the 10 in. singlet lens Halle filter, 5 in. image, about $1 / 30 \mathrm{~S}$ exposure.

and transverse to the neutral line. In earlier low resolution pictures these were not resolved and sometimes confused with filaments.

E. Chromospheric threads (6) (Foukal, 1971) are horizontal features following field lines connecting distant vertical fields of opposite polarity. Along with the FTA, they are only seen in the line center. The threads account for the vortex structure first discovered by Hale et al. (1919). They mark the transverse field direction further out from the active region and also help establish field transitions. Since all flux lines first emerge at a single point, the threads reflect the field history. The thread systems are usually on the edge and the stretching as their sources separate must be responsible for their shallow horizontal structure. On Figure 2, note the $f$ sources (6b) of two sets of threads running to the $p$ spot.

F. Filaments (7) (prominences) lie in channels of horizontal fields (Veeder and Zirin, 1970; Foukal, 1970). They separate regions of opposite polarity, but the field lines run an angle between 0 and $45^{\circ}$ to the boundary. Thus there is a shear in the field, and quite distant sibling fields are connected by the field lines. The prominence $7 \mathrm{a}$ is a typical boundary filament, marking the perimeter of the active region and separating the $f$ part from an extensive $p$ unipolar region. The active region filaments 


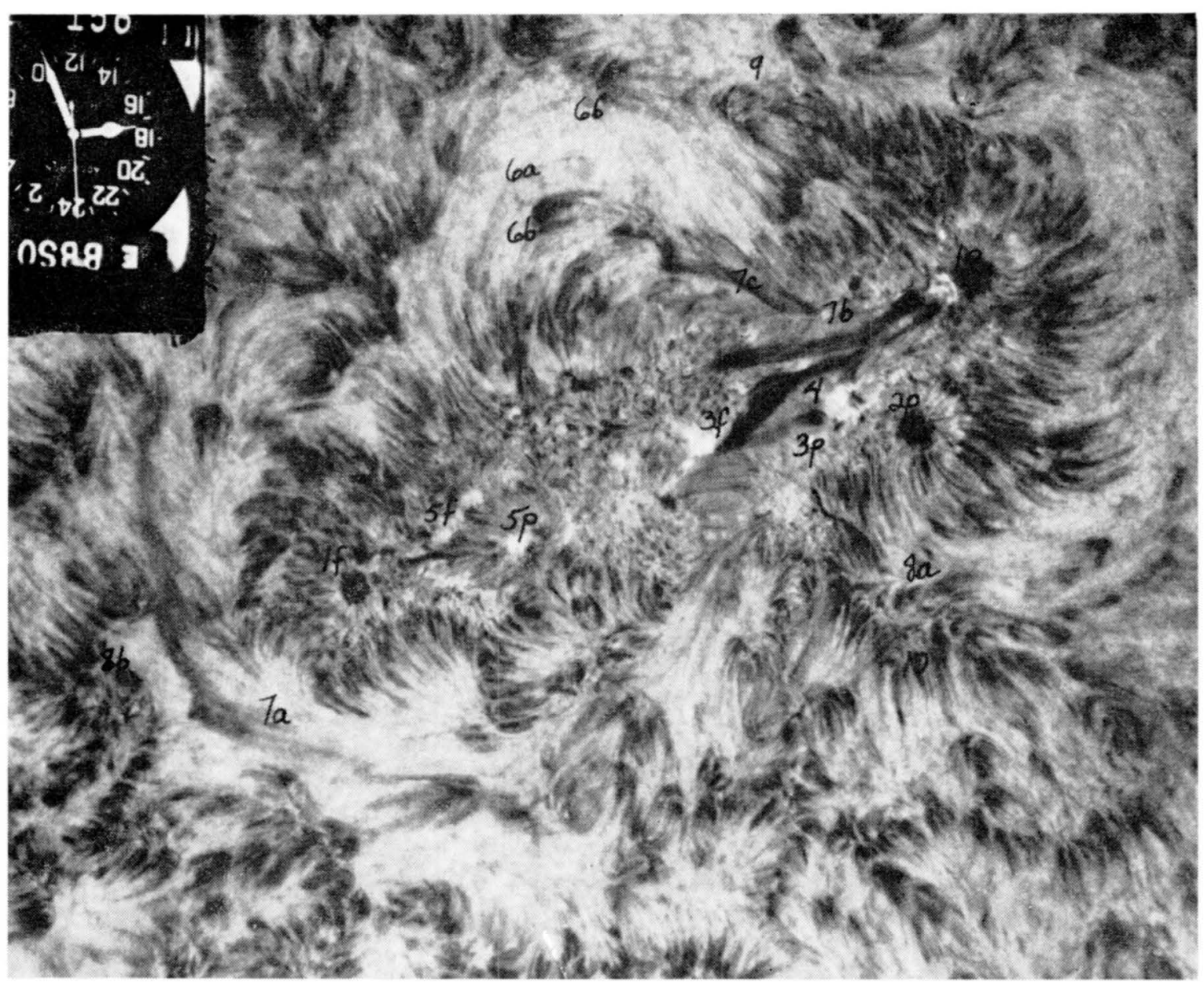

Fig. 2. Same region and time as in Figure $1, \mathrm{H} \alpha+0.5 \AA$. Numbers are explained in the text.

$7 \mathrm{~b}$ and $7 \mathrm{c}$ follow the stretched force lines of the EFR. Such prominences often occur when the EFR is a few days old; they are visible off-band and are fairly high (up to $20000 \mathrm{~km}$ ), and eventually decay to low FTA system. These prominences obviously do not run parallel to the neutral line.

G. Quiet Chromosphere. The quiet chromosphere consists of two classes of region, enhanced network and quiet chromosphere. In the former (Veeder and Zirin, 1970), strong magnetic fields of one polarity form a complete network extending outward from the active regions. In the latter, the bits of field are more ragged, not forming a complete network, but the regions are unipolar. As has been shown by many authors, stronger field is localized in regions that are bright in on-band $\mathrm{H} \alpha$ and at the base of dark spicules off-band. Examination of K-line spectroheliograms in Selected Spectroheliograms (Title, 1969) shows detailed correspondence between $\mathrm{H} \alpha$ and $\mathrm{K}$ emission.* The K-line pictures show additional weak emission corresponding to dark off-band features inside the network. In these clumps of field (such as (8)) we may also have polarity changes marked by dark fibrils, so they are not completely unipolar.

* Note added in proof: Extensive simultaneous cinematography in $\mathrm{H} \alpha$ and Ca II $\mathrm{K}$ carried out in 1971 confirms this result, except that in the active regions there often are bright regions in $\mathbf{K}$, presumably with strong fields, which are covered by dark $\mathrm{H} \alpha$ fibrils and hence not seen, but which are visible off-band as regions of fine granular dark structure. 
The dark spicules (10) follow the lines of force connecting to some distant region of opposite polarity (Foukal, 1970). Since these cannot connect to their neighbors they must go over the top and are more vertical. Foukal has pointed out a test by which these features help us establish the existence of a horizontal field region dividing opposite polarities, but running parallel to the neutral line. Spicules entering such a region from $\mathrm{N}$ polarity will turn one way and those entering from $\mathrm{S}$ will go the other way. The Foukal test easily establishes the existence of the neutral line in the lower left (near 7a) of Figure 2. It is interesting that the fibril and field structure at such boundaries of extensive unipolar regions always run along the boundary; apparently they are sheared by large scale motions and the fields are not strong enough to resist this. At (9) above we see a small $f$ spot connected to the $p$ structure by fibrils; these start out as bright, elongated structures, turn over and collect horizontally.

\section{The Magnetic Field in McMath 19079}

We may now discuss the magnetic structure of Mt. Wilson $18157+18161$ as seen in Figures 1 and 2. We do not have fine magnetograph data, but the field structure is simple and instructive. The history of the group is also interesting and reflected in the field. Mt. Wilson 18157 had been a peaceful bipolar region with a $p$ spot $(2 p)$ and $f$ plage. On October 9 an emerging flux region appeared in the $\mathrm{N}$ half of the region, marked by dark arched filaments, and a new $p$ and $f$ spot appeared. These rapidly drew apart although we have no observations on October 10, we can be sure that the $p$ spot $1 p$ in Figure 1 is that first seen on the 9th; and probably the $f$ spot sibling* has broken up into several spots in the plage $3 f$. The long dark active region prominences $7 \mathrm{~b}$ and $7 \mathrm{c}$ mark field lines which connect the two poles of the EFR and have been stretched by the rapid separation of the two halves, while the threads (6) similarly connect outlying $p$ and $f$ polarity. On the other hand the FTA system (4) connects the extended plages. It has been bent by the flow of the spot $1 p$ past $2 p$. The connection of the $p$ and $f$ plages is much like the meeting of two large delegations; only those at the interface may shake hands, while those in the back rows must reach over the top or around the sides to greet their counter parts. In the same way, only neighboring fields may be connected by field transition arches, and all other field lines must go around or over the top. We may reasonable assume that there is a system of arched lines of force above the surface connecting the plages. As mentioned above (5) is an EFR in the middle of an $f$ plage. Three $p$ spots (as can be seen in the off-band picture Figure 2) at $5 p$ are connected by FTA's to the plage following them, but some of the field lines are connected to the plage in front. Since EFR always emerge as bipolar flux loops, we can only surmise that some field lines have reconnected the $p$ spots to the $f$ plage ahead, rather than their $f$ siblings to the east. This may be responsible for the brightness at the bottom of the field transition arches, as the magnetic energy is released. These spots are erroneously marked $f$ on the Mt. Wilson drawing for the day, but are

* We shall use the term 'sibling spots' for $f$ and $p$ spots born in the same emerging flux region, now more widely separated, but still connected by some lines of force. 
correctly marked $p$ on the Rome drawing. The large following spot $l f$ is an anomaly. It may be connected with the EFR (5), but without observations October 10 we cannot tell this. This spot weakened considerably on the 11th and disappeared on the 12th. Large $f$ spots like this without a $p$ sibling are uncommon.

The brightness variation as a function of magnetic field intensity and growth gradient is of great significance to the understanding of plages. The intensely bright plage at the base of the field transition arches (4) is characteristic of emerging flux regions; so long as it is bright we know empirically that field continues to emerge; there is a well-defined (but unknown) mechanism by which emerging flux heats the chromosphere. In a stable plage there is no special brightening at the foot of FTA.

The unique and inviolable (except for sunspots themselves) connection between brightness and the presence of vertical field tells us that the chromosphere is only heated where the field is vertical. Movies show a continuous brightness oscillation in the elements of the plage*. These elements are smaller than photospheric granulation (typically they seem to be $500 \mathrm{~km}$ across and show some complex structure). J. W. Evans (private communication) has called them 'crochets' in view of an occasional hook or looped structure. The structures are just at the limit of resolution. The matter is puzzling, because one would expect emerging vertical field lines to be marked by point structures; on the other hand the field lines bend over sharply after they emerge, and this could explain the hook-shaped appearance. It is obvious that the exact nature of these structures is basic to the theory of plages and the connection between vertical field and brightening.

In the upper right of Figure 2 (SE) we see a beautiful example of the Foukal test that fibrils curve oppositely on either side of a filament. All the dark fibrils (or spicules) in the SE corner point away from the filament, except those near it, which point to the lower right. On the opposite side of the filament (near the $f$ spot) the fibrils all point in the opposite direction. The only exception is the little bush marked $8 p$ which points the other way and proves under closer scrutiny to be a little $p$ spot.

\section{Sunspot Development}

In our pictures we see examples of late stages in the development of emerging flux regions. Weart and Zirin (1969) showed that these are the unique form by which new sunspot groups emerge. Zirin (1970c) discussed a growth pattern for large groups in which $p$ and $f$ spots rapidly separate (in the classic manner observed by many), stabilize, and then rise to activity in a second stage of development. Examination of many cases observed at Big Bear shows that all new sunspot flux emerges in strictly bipolar form as EFR's. The subsequent development of complex regions results from the reconnection of lines of force and distortion of the expanding EFR's as they reach equilibrium with the older spot groups. We have considerable evidence for such field reconnection which will be presented in a later paper; often this is connected with

* These have now been measured photometrically by Bhatnagar and Tanaka and appear to have a $300 \mathrm{~s}$ period. 
flares. The EFR's invariably expand in longitude, unless they run into other spots.

The picture of these expanding dipoles of course is a very attractive feature of the Babcock-Leighton theory of erupting flux loops. The pair of spots would be the intersection with the surface of a large emerging arched tube of flux. As the tube rises, the intersection separates. Obviously every line of force must have two ends, so the new flux must always be bipolar. There are several problems with this picture: first, the sunspots always are the outermost extremity of the rising arch, with no precursor of lower field strength. Perhaps the outer fields have been swept up in the motion but then why are the penumbrae so well formed, with typical stable tulip-shaped fields? One could understand why there is so much stronger field between the spots the field inside the arch has been partly trapped by the conducting material on the surface. But it is rather curious that we see no brightening at all at the outer edge of the expanding spot pair, while the interior is extremely bright. In Figure 1 we can see no particular distinction between the old spot la and the expanding one $1 \mathrm{~b}$, except for the arches and fibrils connecting the latter to its sibling. The problem is most intriguing.

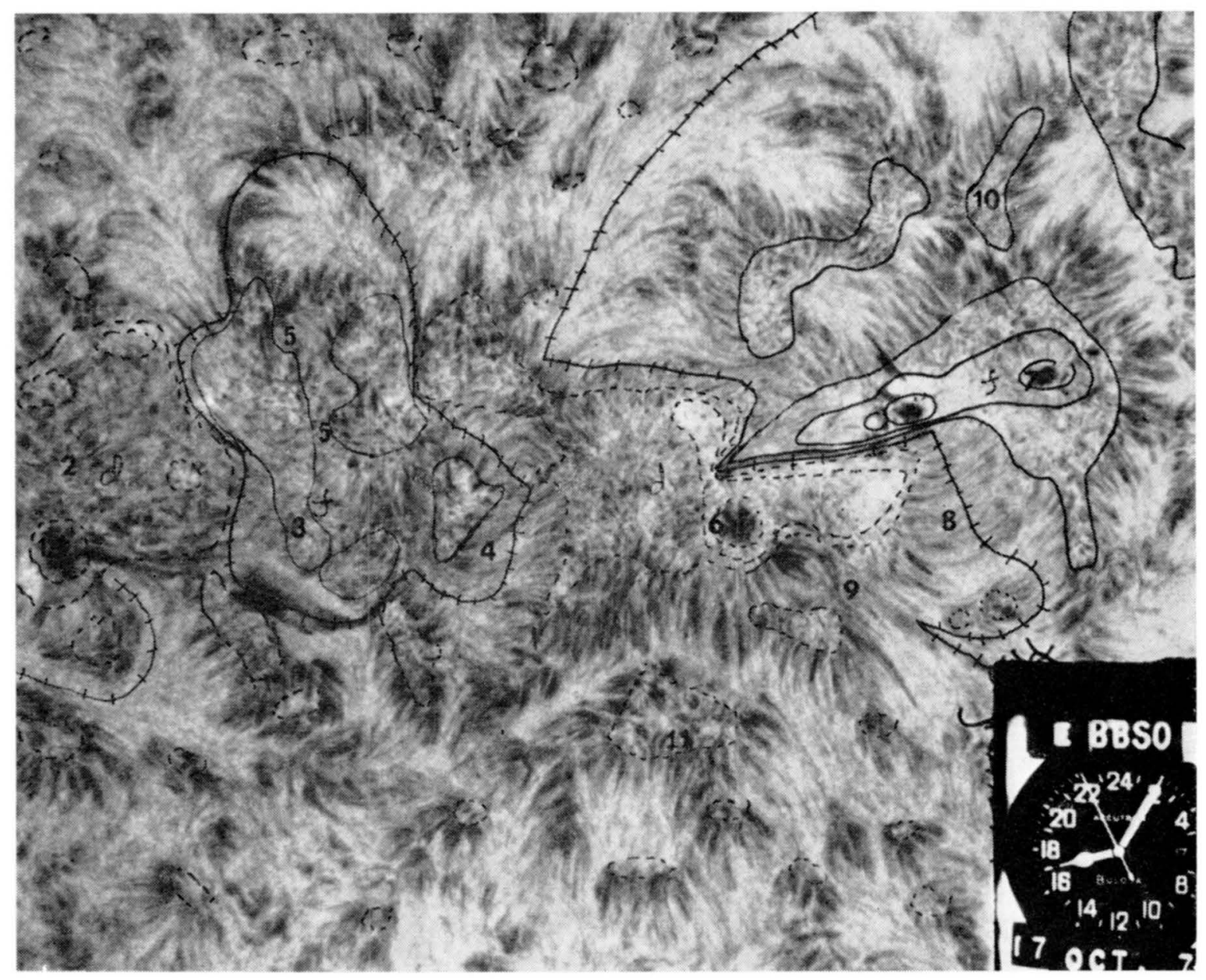

Fig. 3a. $\mathrm{H} \alpha+\frac{1}{2} \AA$, Big Bear filtergram, October 17, 1970, 1705 UT. The dashed contours are preceding polarity; solid are following; the cross-hatched solid line marks the longitudinal neutral line. These contours were drawn solely from the $\mathrm{H} \alpha$ and a copy sent to Dr Harvey before he sent us the magnetogram. S top, W left. 


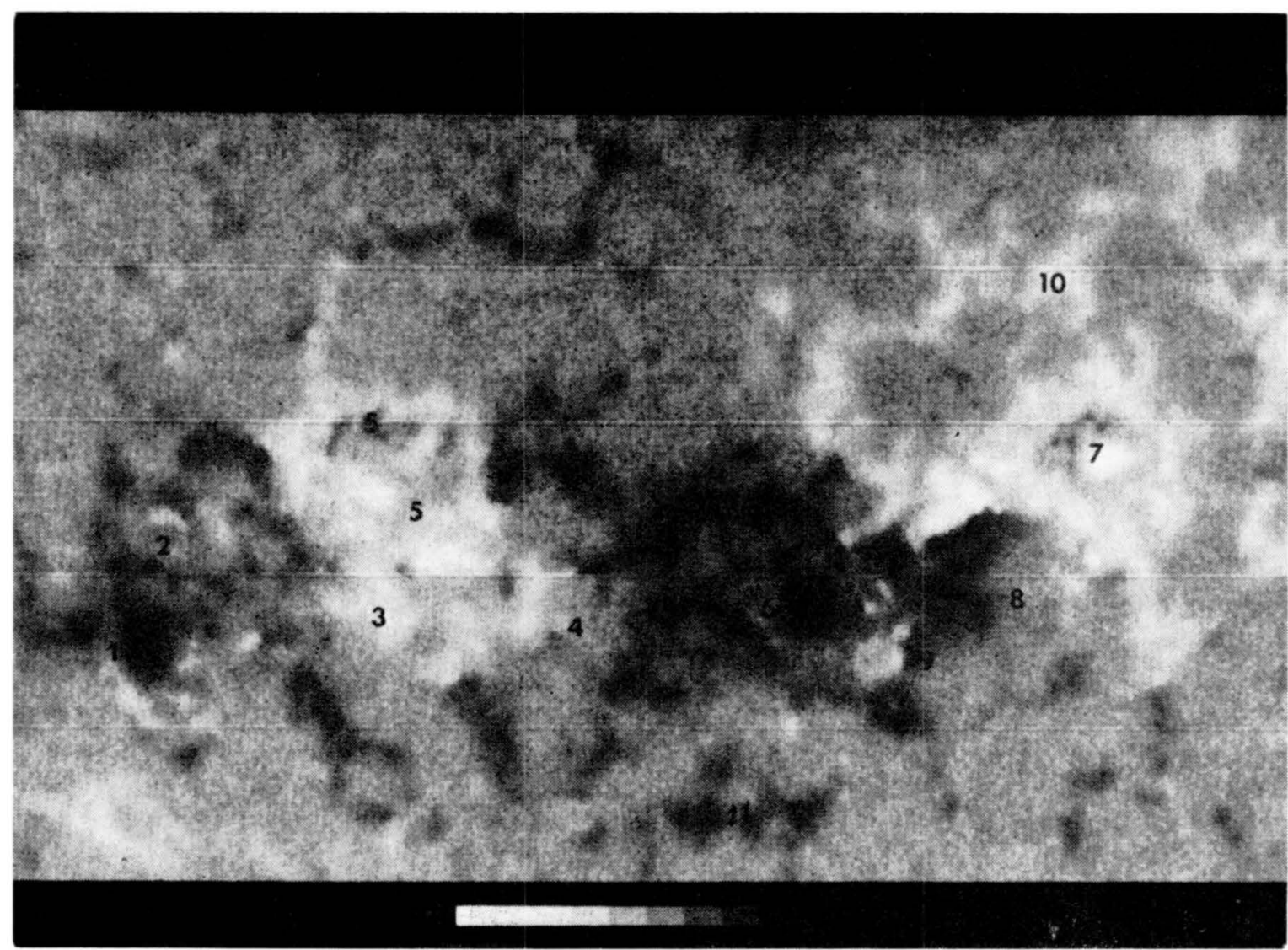

Fig. 3b. Magnetogram obtained by J. W. and K. Harvey, 1502 UT, at Kitt Peak. Dark polarity is preceding, bright following. The magnetogram is calibrated for five discrete levels of field intensity 0 to \pm 25 , to \pm 220 , to \pm 620 , to \pm 1220 , to $2000 \mathrm{G}$, and the spatial resolution is close to 2 arc sec.

We shall see below that weak flux also erupts in bipolar form - but somewhat less regularly than the spot fields.

\section{Comparison with High-Resolution Magnetograms}

In order to establish our ability to deduce magnetic fields from $\mathrm{H} \alpha$ filtergrams, we conducted a test in October 1970: we used an excellent high resolution sequence on October 16-17, 1970, to predict magnetic field maps of the same region obtained by Dr John W. Harvey and Karen Harvey with the Kitt Peak multichannel magnetograph (Livingston, 1968). Without even examining Mt. Wilson spot drawings we produced the magnetic field map shown in Figure 3, superposed on the off-band Big Bear picture.* This map was sent to Dr Harvey, who then sent us his magnetogram (of course he did not try to predict the $\mathrm{H} \alpha$ picture, because he did not have transverse field measurements).

* This procedure is far more difficult than just comparing post facto the magnetogram and filtergram, but of course it is the only objective test. For a complex region, it is as time-consuming as just making a magnetogram, but forces the analyzer to understand the chromospheric structure. And one gets the transverse fields as well. 


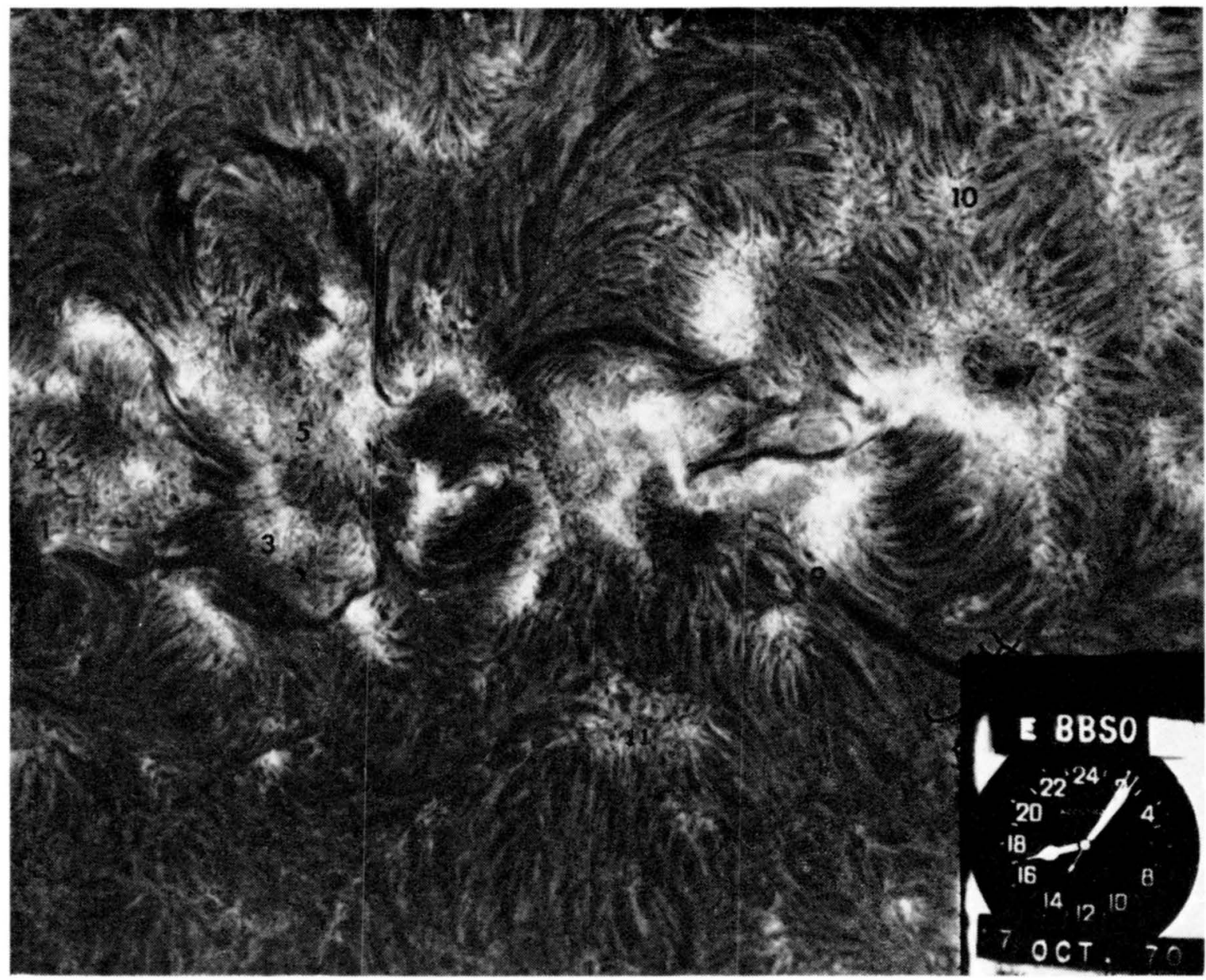

Fig. 4. H $\alpha$ centerline photo, 1629 UT, October 17, 1970, with features numbered and explained in the text. S top, W left.

Field determination was particularly difficult in this case because the region in question (Mt. Wilson 18164-5-9) was old and very complex. It appeared as an EFR on September 21, with $\mathrm{N}-\mathrm{S}$ lines of polarity, straightened out in the next two days to the normal orientation and rotated off as a moderately complex bipolar group. When it reappeared it was quite weak, but two EFR's appeared in the preceding and following parts of the region. This led to complicated structure which made field determination difficult, but the results are fairly good. Figure 3 a shows the magnetogram from Kitt Peak, kindly reproduced by Dr Harvey to the same scale as Figure 3b, which shows the off-band Big Bear picture with inferred magnetic contours drawn on it. By and large the agreement is excellent; the polarities are generally correct, and the peak fields are in the same place as the peak $\mathrm{H} \alpha$ emission. The principal discrepancy is in the presence of numerous small areas of fields of opposite polarity imbedded in larger regions of moderate field. The existence of such regions was unknown to us; however, when we re-examined the $\mathrm{H} \alpha$ pictures, we found that such regions are usually marked, particularly off-band, by dark fibrils connected with the field transition. It now should be possible to predict most of these included fields by looking for these fibrils. Another 
failure is that we could not properly draw the neutral line at the upper right (NW) because we did not have the rest of the picture, and the fields in that corner are somewhat weak.

We now describe the region in detail: At left is a preceding spot (1) with an extensive $p$ region below it. This region has a number of bits of $f$ polarity in it such as at (2). There also is $f$ polarity directly $\mathbf{N}$ of the $p$ spot; these are barely detectable by dark fibrils present. The neutral line above the spot marks an open channel - the fields above it are all $f$ (incorrectly predicted $p$ ). The sunspots in this picture are all complex and ragged, with numerous light bridges. The $p$ spot (1) is connected by a filament and a set of field transition arches (FTA) to its sibling following polarity region (3) behind. This large following region is bounded by well-developed filaments and by another set of FTA (4). Inside it, particularly in the lower part, are several bits of $p$ polarity (5) marked by dark fibrils. These are remnants of spots which faded out the preceding day. Another $p$ region follows; the $p$ spot (6) and the following spot (7) were siblings in an EFR; they now are connected by one or two long fibrils which form part of the FTA system. The complex region between them marks the area through which they have spread apart. As the region develops and the fields spread out in latitude, these should form the normal FTA systems.

Both spots (6) and (7) have regions of opposite polarity near them which we missed but which can be detected in retrospect by dark fibrils. These spots are typical of the satellite fields of opposite polarity which have been discussed by Sheeley (1969) and Vrabec (1971) observed that they are moving outward from the main spot. On our films we cannot detect any outward motion.

Another region of included opposite polarity appears at (9); it is easy to pick out after seeing the magnetograms by the FTA's leading from spot (6). Note also that the FTA system (8) clearly marks the horizontal field between the plage regions surrounding (6) and (7), and the neutral line then joins to the filament extending from (9).

I beseech the reader to study these pictures carefully; they contain a wealth of information about the Sun.

\section{Quiet Regions}

The rules for quiet regions are the same as for active, but quiet regions have their own characteristic structures. Figures 3 and 4 contain more enhanced network; with the fine magnetogram we may test some of the assertions of Section 3. The presence of longitudinal field is marked by emission in the line center, and fine granular structure off-band, very similar to the structure in the weaker plages. On the off-band picture (Figure 4) we may see photospheric granulation inside the network cells; the $\mathrm{H} \alpha$ granular structure in the plagettes is smaller, confined, no doubt, by the magnetic field.

From each plagette, a 'bush' of spicules protrudes, usually pointing along field lines in the direction of lower field strength. At the bottom of the frame we see how the spicules apparently reach over the top of their neighbor, connecting with quite distant regions of the opposite polarity out of the picture. After Cragg et al. (1963) recognized that spicules are concentrated around plagettes, Beckers (1964) pointed 
out the existence of herringbone structures such as that around the plagette (10). The only difference between this and the normal 'bush' is that the plagette is elongated in a direction outward from the center of the active region, so that the spicules tend to bend outward in two directions.

In cine projection many dark spicules move up and down like jets, but others are longer lived and wave around with the general oscillation. Apparently the curving magnetic field lines coming out of the plagette align the denser structures, which may be longer lived condensations or may be spicule jets. The features are most complex and just at the limit of resolution, about $500 \mathrm{~km}$.

The plagettes of the enhanced network are not strictly unipolar but include some opposite field. Plagettes like (11) have the polarity of the unipolar region and spicules that point along the field; but they also show internal structure characteristics of field reversal, namely small dark horizontal fibrils. The magnetogram clearly shows the field reversal. When followed in time these regions are seen to arise from below in bipolar form with a modest amount of activity (little surges, small dark eruptions), marking them as places where weaker field has erupted and is making its peace with the surrounding fields by reconnection. But they are qualitatively different from the EFR's.

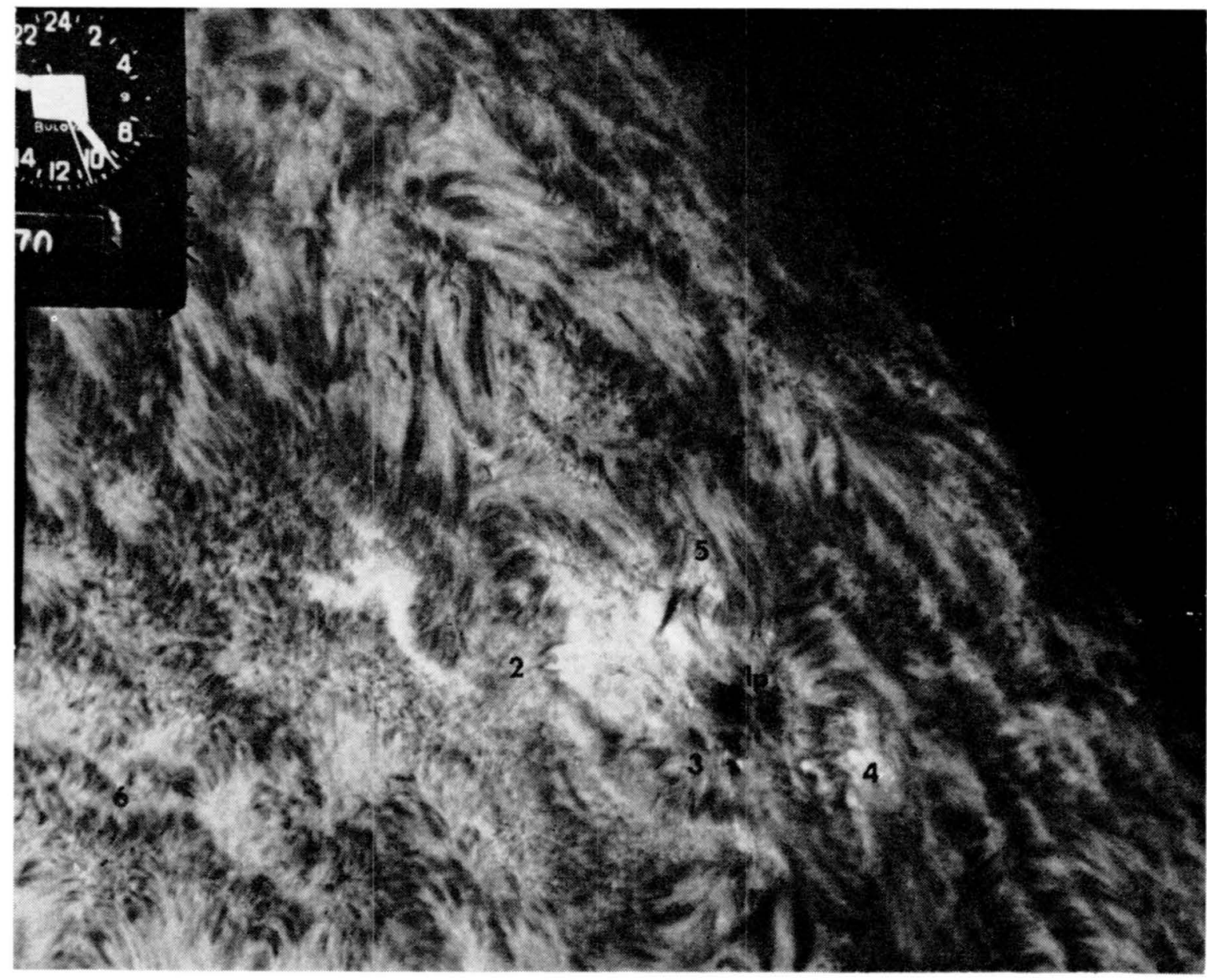

Fig. 5. H $\alpha$ centerline September 9, 1970, with magnetic features marked. N top, E left. 


\section{A Region near the Limb}

Our third example is the spotgroup, Mt. Wilson 18096. We show H $\alpha$ (Figure 5) and $\mathrm{H} \alpha+\frac{1}{2} \AA$ (Figure 6). Mt. Wilson fine scan magnetograms and Big Bear $\mathrm{H} \alpha$ are available for the period September 5-8; they generally confirm the fields derived from $\mathrm{H} \alpha$, but they are not so fine as the pictures on the 9 th. The region is dominated by a complex group of $p$ spots $(1 p)$, followed by bright $p$ plage. These are separated by well-developed FTA system (2) followed by an extensive $f$ plage. Because the region is close to the limb we get some idea of the vertical structure in it. For example, just below the numeral (2) we see dark fibrils arching over the bright plage. The same is true of (3), where a $p$ penumbra projects into the $f$ plage. The extensive FTA system grew up in a few days as the result of the emergence of numerous bipolar EFR spots in the following plage, which eventually merged into the system seen. Figure 7 shows the region on September 3, before the new fields had emerged. The FTA system was small and the $p$ plage was non-existent. There were two filaments $\mathrm{E}$ and $\mathrm{N}$ of the spots, which blew off on the 5th and 7 th as new flux erupted. The entire development of the $p$ plage occurred on the 7 th and 8 th, accompanied by rotation of the two big $p$

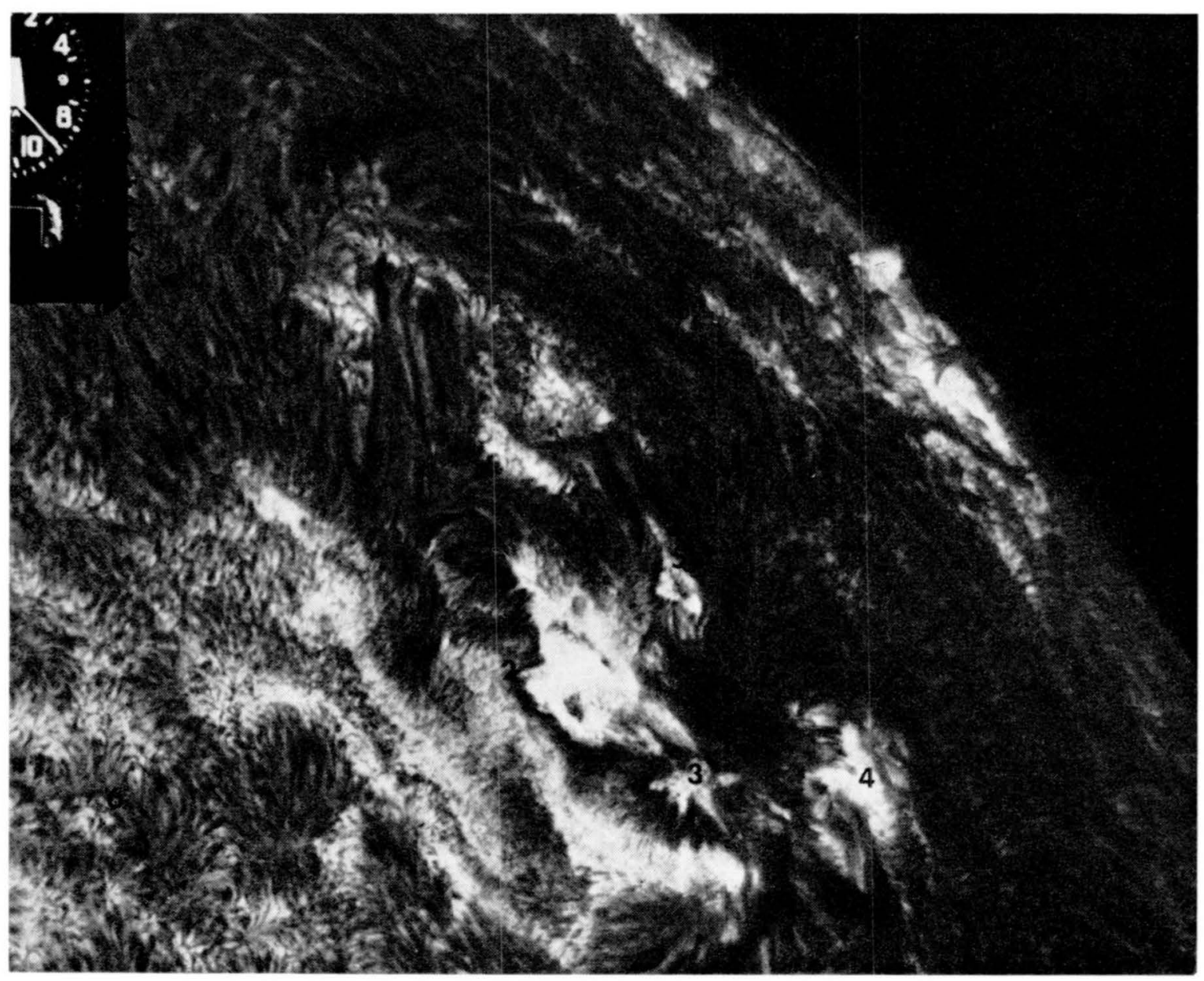

Fig. 6. Same as Figure 5, $\mathrm{H} \alpha-0.6 \AA$. 
spots through $90^{\circ}$ and a considerable decrease in the size of the northern, elongated umbra.

I have remarked (Zirin, 1970c) on the fact that flares tend to occur in regions of inverted polarity ahead of the main spot. Two such regions appear here at (4) and (5) In each case there is a bright EFR with the $f$ polarity leading. The reversal may be deduced from the fact that the front of each bright region connects to the main $p$ spots

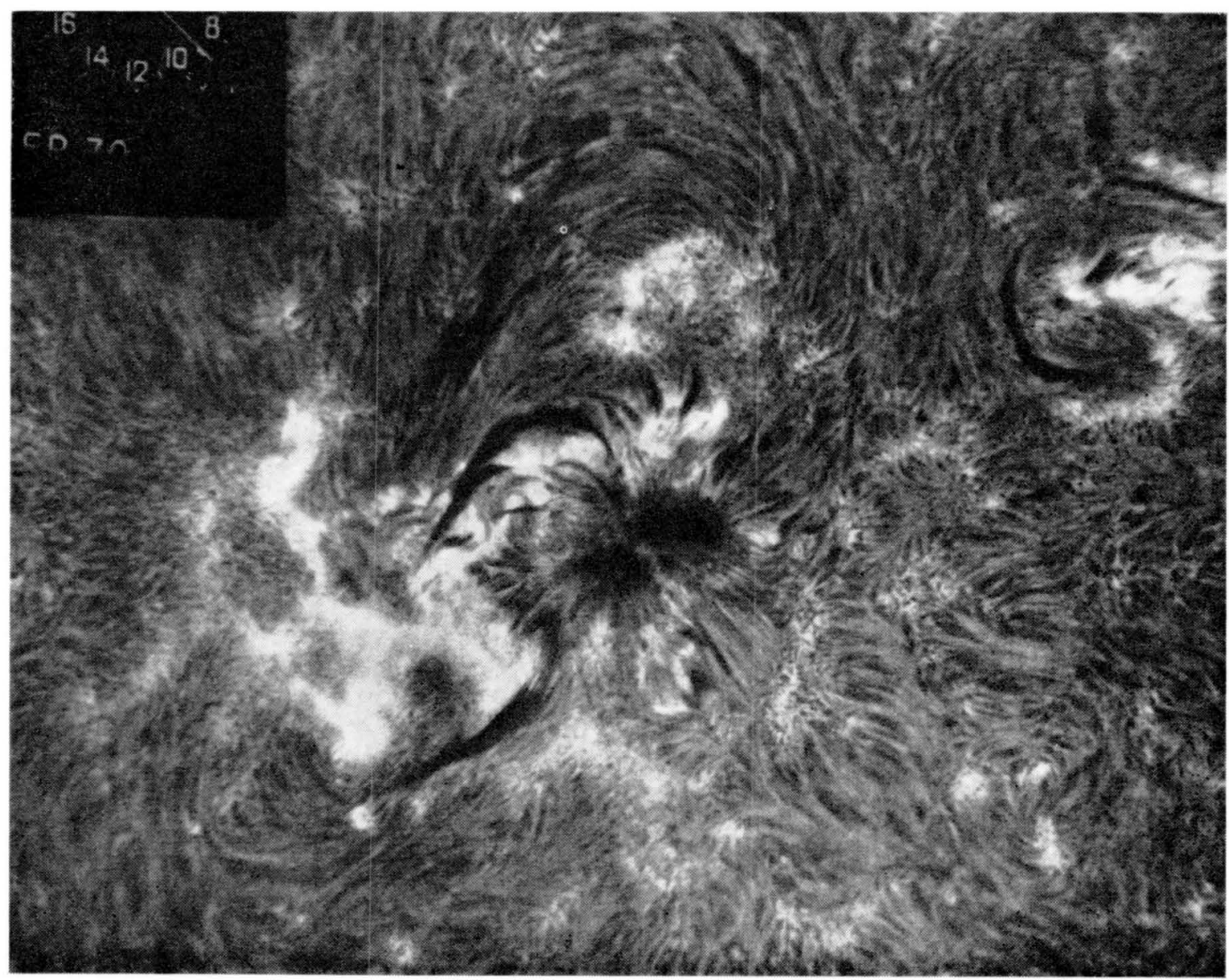

Fig. 7. Same region as Figures 5 and 6, September 7, 1970.

by dark fibrils, while the $\mathrm{E}$ part (which is $p$ ) belongs to the general $p$ plage. These regions were small but they did produce most of the limited activity in the region. In my earlier paper (Zirin, 1970) I considered the included $f$ polarity alone; but clearly magnetic field can only erupt in bipolar form in the EFR. Of course, on the low resolution Mt. Wilson magnetograms the small $p$ spot merges with the larger $p$ field and we are left with the $f$ plage and inverted polarity. The lines of force connecting the $f$ spot to its sibling have reconnected to the main $p$ spot. Field line reconnection is thus an energy source for the flares.

Ultimately, the source of this energy is the buoyancy which lifted the new EFR to 
the surface, thereby displacing the field lines of the original spot. Field line reconnection puts the field in a lower energy state. Why wasn't the EFR pushed away by the existing field as it rose? Although there is no statistical evidence available at the moment, experience shows that these little bipolar fields have a strong tendency to erupt near established $p$ spots; there is thus a force which brings them there, and ultimately shows up in the flare energy, or that channels the buoyancy energy into this task.

A particularly interesting feature of the development of this group is the growth of the extensive following unipolar region, limited at first, but covering $30^{\circ}$ of longitude at the end. The plagettes in this region do not appear to break away from the following plage, but emerge in complex new plagettes. Since all new flux must be bipolar, these must shed the $p$ part of their flux and make up the large unipolar region.

Flux must erupt in bipolar form; there is apparently some process which cancels part of this flux with the main plage leaving the 'dominant' polarity in the unipolar region. If the plagette is still bipolar, lines of force associated with the fibril and spicule structure would return to the surface, rather than appear to connect to distant regions. Such recurvature is in fact observed at point (6). We thus have the possibility of detecting the origin of an element of flux by whether the spicule structure is predominantly vertical, connecting to distant points (implying that it is a remnant of the original sunspot) or curving back to the surface, implying local origin. Better picture sequences are needed.

\section{Conclusion}

We have tried to understand the meaning of $\mathrm{H} \alpha$ morphology and its connection with the magnetic field structure. There is a remarkable separation into bright regions of nearly vertical field, where the field apparently facilitates the energy transport to the chromosphere, and dark regions of nearly horizontal field where such transport is suppressed. The dark fibrils invariably parallel the magnetic field lines and therefore show us the direction of transverse fields. All magnetic flux emerges in bipolar form, closely space; the bipolar units are stretched as flux loops emerge and the spots separate; they are further affected by crowding by other emerging fields and possibly differential rotation. Since most of the stretching is horizontal, field lines in active centers tend to be flat and low, while those connecting unipolar regions will be more of the arched shape. Strong plages of opposite polarity are separated by sets of field transition arches, with field lines running across the boundary, while weaker, more extensive fields are separated by channels of long horizontal fibrils running more or less parallel to the boundary. When the conditions are right, such a channel will be occupied by a prominence. When new flux erupts in such a channel, the prominence is unstable and erupts. For this reason, any prominence with a plage underneath it is unstable and will erupt.

When new bipolar flux erupts near an existing spot, or when the expansion of a bipolar flux loop brings unrelated fields together, field lines reconnect to form lower energy configurations and flares result. 


\section{Acknowledgements}

I am grateful to Dr J. W. Harvey and Mrs. Harvey for their magnetogram, to Dr Dale Vrabec and Dr Robert Howard for their magnetograms, and to Drs P. Foukal and S. Prata for numerous discussions. The work was supported by NSF under Grant Ga. 24015 and NASA under Grant NGL 05002034.

\section{References}

Beckers, J. M.: 1964, Sacramento Peak Observatory Contributions, No. 75, Ph.D. Thesis. Bruzek, A.: 1967, Solar Phys. $2,451$.

Bumba, V. and Howard, R : 1966, Astrophys. J. 143, 592.

Cragg, T., Howard, R., and Zirin, H.: 1963, Astrophys. J. 138, 303.

Foukal, P.: 1970, Solar Phys. 13, 330.

Foukal, P.: 1971, Solar Phys., 19, 59.

Hale, G. E., Ellerman, F., Nicholson, S. B., and Jay, A. H.: 1919, Astrophys. J. 49, 153.

Howard, R. and Harvey, J. : 1964, Astrophys. J. 139, 1328-1335.

Livingston, W. C.: 1968, Astrophys. J. 153, 929.

Prata, S.: 1971, Solar Phys., 20, 310.

Sheeley, N.: 1969, Solar Phys. 9, 347.

Veeder, G. J. and Zirin, H. : 1970, Solar Phys. 12, 391.

Vrabec, D.: 1971, in R. Howard (ed.), 'Solar Magnetic Fields', IAU Symp. 43, 329.

Vrabec, D.: 1971 (private communication).

Weart, S. and Zirin, H. : 1969, Publ. Astron. Soc. Pac. 81, 270.

Zirin, H.: 1970a, Phil. Trans. Roy. Soc. London A270, 77.

Zirin, H.: 1970b, in R. Howard (ed.), 'Solar Magnetic Fields', IAU Symp. 43, 237.

Zirin, H. : 1970c, Solar Phys. 14, 328. 